\title{
Baryons and Skyrmions in QCD with Quarks in Higher Representations
}

\author{
Stefano Bolognesi * \\ The Niels Bohr Institute, Blegdamsvej 17, DK-2100 Copenhagen Ø, Denmark
}

\begin{abstract}
We study the baryonic sector of QCD with quarks in the two index symmetric or antisymmetric representation. The minimal gauge invariant state that carries baryon number cannot be identified with the Skyrmion of the low energy chiral effective Lagrangian. Mass, statistics and baryon number do not match. We carefully investigate the properties of the minimal baryon in the large $N$ limit and we find that it is unstable under formation of bound states with higher baryonic number. These states match exactly with the properties of the Skyrmion of the effective Lagrangian.
\end{abstract}

December, 2006

*bolognesi@nbi.dk 


\section{Introduction}

The large $N$ expansion is a major tool in the study of strongly coupled $S U(N)$ gauge theories [1]. In the double line notation gluons are represented by two lines with opposite oriented arrows and quarks, if they are in the fundamental representation, they are represented by a single oriented line. To every Feynman diagram there corresponds a certain topological oriented surface with a certain number of handles and holes. Holes correspond to quark loops. Every handle suppresses the diagram by a factor $N^{-2}$ and every hole by a factor of $N^{-1}$. The large $N$ limit is thus dominated by diagrams with only planar gluons and fermion quantum effects are only present in the subleading orders.

Under the weak assumption of confinement for arbitrary large $N$, a lot of physical information can be inferred from the large $N$ limit even if the resummation of the planar gluons is not possible [2]. Of particular interest for what follows are the baryons whose gauge wave function is

$$
\epsilon_{\alpha_{1} \ldots \alpha_{N}} Q^{\alpha_{1}} \ldots Q^{\alpha_{N}}
$$

It is a gauge singlet completely antisymmetric under exchange of two quarks. The antisymmetric property of the gauge wave function (1.1) implies that the spatial wave function is symmetric under exchange of quarks. In the large $N$ limit the baryon can be approximated as a system of free bosons confined in a mean potential. The mass of the Bose-Einstein condensate scales like the number of particles $N$. An important property of baryons in the large $N$ limit is that they can be identified with the solitons of the chiral effective Lagrangian [3, 4].

The large $N$ limit with a fixed number of quarks in the fundamental representation, has the disadvantages that all the quantum corrections due to quark loops vanishes as $\frac{1}{N}$. For example the $\eta^{\prime}$ mass vanishes like $\frac{1}{N}$ since its value comes only from the axial $U(1)_{\mathrm{A}}$ anomaly. In order to cure these kinds of problems another kind of limit has been suggested in the past, where the number of fundamental quarks $N_{f}$ is send to infinity keeping fixed the ratio $\frac{N_{f}}{N}$ [7]. Although phenomenologically appealing, this kind of limit is even more difficult to solve than the original 't Hooft limit.

Recently another kind of large $N$ limit has received considerable attention. This is the case of quarks in the two index, symmetric or antisymmetric (S/A), representation. Armoni, Shifman and Veneziano have discovered that a theory with $N_{f}$ 
Dirac quarks in the two index S/A representation is equivalent, in a certain bosonic subsector and in the large $N$ limit, to a theory with $N_{f}$ Weyl quarks in the adjoint representation [9]. Particularly interesting is the antisymmetric representation since it can be used to reproduce QCD at $N=31.1$. This equivalence becomes particularly useful when $N_{f}=1$ since the theory with one fermion in the adjoint is $\mathcal{N}=1$ super Yang-Mills and some non-pertubative results are known about it. This has been used in [11] to make quantitative predictions about QCD.

Now we will face the central issue of this paper. It has been noted in [10] that, at least at a first glance, the identification between baryons and Skyrmions in the large $N$ limit does not work. A natural choice for the gauge wave function of the baryon is the following

$$
\epsilon_{\alpha_{1} \alpha_{2} \ldots \alpha_{N}} \epsilon_{\beta_{1} \beta_{2} \ldots \beta_{N}} Q^{\alpha_{1} \beta_{1}} Q^{\alpha_{2} \beta_{2}} \ldots Q^{\alpha_{N} \beta_{N}}
$$

where the formula holds for both the symmetric and antisymmetric representations. This baryon is formed of $N$ quarks and so the first guess is that its mass scales likes $N$ in the large $N$ limit. The mass of the Skyrmion scales like $F_{\pi}^{2}$ where $F_{\pi}$ is the pion decay constant. In the case of the quarks in higher representations $F_{\pi}$ scales like $N$, so the mass of the Skyrmion scales like $N^{2}$ in contrast with the naive expectation for the baryon (1.2). This is the puzzle we are going to solve in this paper.

The first step towards the solution is to realize that the naive expectation that the mass of (1.2) scales like $N$ is not correct. The reason is the following. The gauge wave function (1.2) is symmetric under exchange of two quarks. Since the total wave function must be antisymmetric, this means that the space wave function must be antisymmetric (this has also been noted in [12]). The large $N$ baryon must thus be approximated as a set free fermions in a mean field potential. Since fermions cannot all be in the same ground state, there is an extra term in the energy coming from the Fermi zero temperature pressure. At this point one could hope that this extra term could compensate the mismatch and make the baryon mass scale like $N^{2}$. A detailed analysis shows that this is not true.

Another problem for the candidate baryon (1.2) comes from the Wess-ZuminoWitten term of the effective Lagrangian for the Nambu-Goldstone bosons [5, 6]. From this term we can read off the statistics and the baryon number of the Skyrmion. The baryon number is $\frac{N(N \pm 1)}{2}$, where \pm stands respectively for symmetric and antisym-

\footnotetext{
${ }^{1}$ The idea of using quarks in the two index antisymmetric representation to reproduce QCD at $N=3$ where first considered in 8
} 
metric representation, and the statistics is fermionic or bosonic accordingly if $\frac{N(N \pm 1)}{2}$ is odd or even. There is no way to recover this numbers from the baryon (1.2).

The topological stability of the Skyrmion in the effective Lagrangian indicates that, at least in the large $N$ limit, there should exist a stable state composed by $\frac{N(N \pm 1)}{2}$ quarks and whose mass scales like $N^{2}$. This is possible if there exist a color singlet wave function that not only is composed by $\frac{N(N \pm 1)}{2}$ quarks, but is also completely antisymmetric under exchange of them. In this paper we will show that this function exists and that $\frac{N(N \pm 1)}{2}$ is the minimal amount of quarks needed for its existence. This also confirm the stability of these baryons. In fact any baryonic particle with a smaller number of quarks must have the extra contributions to its mass coming from the spatial Fermi statistics.

The case of the antisymmetric representation and $N$ odd is particularly interesting. In this case we can show that the baryon (1.2) is identically zero and it is also possible to prove that any gauge invariant quantity symmetric under exchange of two quarks vanishes identically.

The paper is organized as follows. In Section 2 we study the effective Lagrangian and the Skyrmion properties. In Section 3 we study baryons at large $N$. In Section 4 we find the stable baryons that can be identified with the Skyrmions in the large $N$ limit. In Section 5 we consider some peculiar property of the antisymmetric representation. Finally in Section [6] we discuss the stability of the Skyrmion.

\section{$1.1 \quad$ Note on conventions}

The conventions we use in the paper are the following: $Q^{\{\alpha \beta\}}$ indicates a quark in the two index symmetric representation while $Q^{[\alpha \beta]}$ indicates a quark the two index antisymmetric representation. When we write $Q^{\{\alpha \beta]}$ it means that the formula we are writing is valid for both the representations. When we formally split the quark $Q^{\{\alpha \beta]}$ into two fundamental quarks we will use the symbols $q^{\alpha}$ and $q^{\beta}$. We reserve the name quark or higher dimensional quark for $Q^{\{\alpha \beta]}$, while we will use the name fundamental quarks for $q^{\alpha}$ and $q^{\beta}$. 


\section{Effective Lagrangians, Anomalies and Skyrmions}

Consider asymptotically free theories with $N$ colors and $N_{f}$ Dirac fermions transforming according to the two index (anti)symmetric representation of QCD

$$
\mathcal{L}=-\frac{1}{2} \operatorname{Tr} F_{\mu \nu} F^{\mu \nu}+\sum_{k=1}^{N_{f}} \bar{Q}_{\{\alpha \beta]}^{k}\left(i D_{\mu} \gamma^{\mu}-m_{k}\right) Q_{k}^{\{\alpha \beta]}
$$

$F_{\mu \nu}=\partial_{\mu} A_{\nu}-\partial_{\nu} A_{\mu}+i g\left[A_{\mu}, A_{\nu}\right]$ is the field strength, $g$ is the coupling constant, and the covariant derivative is $D_{\mu}=\partial_{\mu}-i g A_{\mu}$. In order to have a well defined large $N$ limit we take the product $g^{2} N$ to be finite.

At large $N$ the theory reduces to an infinite tower of weakly coupled hadrons whose interaction strength vanishes like $N^{-2}$. The large $N$ behavior of these theories is similar to that of theories with fermions in the adjoint representation of the gauge group. The dependence of the number of colors for the meson coupling can be evaluated using the planar diagrams presented in Figure 1 and paying attention to the hadron wave function normalization. We will denote the decay constant of the

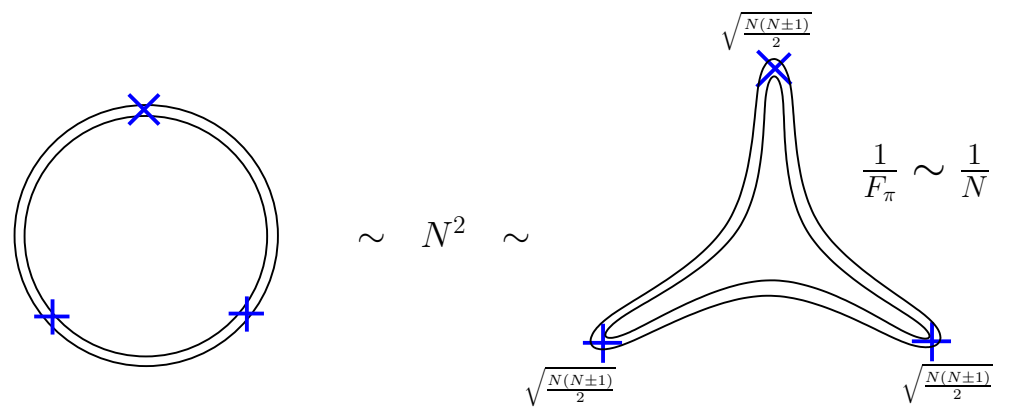

Figure 1: The $N$ dependence of the meson coupling $F_{\pi}$.

typical meson by $F_{\pi}$.

Using a double line notation the Feynman diagrams can be arranged according to the topology of the surface related to the diagram. The $N$ powers of the Feynman diagrams can be read off from two topological properties of the surface: the number of handles and the number of holes. Every handle carries a factor $N^{-2}$ and every hole carries a factor $N^{-1}$. In the ordinary 't Hooft limit where the quarks are taken in the fundamental representation, the holes are given by the quark loops. In the 
higher representation case quarks are represented by double lines as the gluons and so there are no holes but only handles. The contribution to $F_{\pi}$ in the large $N$ limit can thus be arranged as in Figure 2 where the leading order is a quark closed double line with planar quarks and gluons inside, and the next subleading order is given by adding a handle. The leading order scales like $N^{2}$ while the subleading order scales like $N^{0}$. The previous color counting is not affected by the addition of a finite number
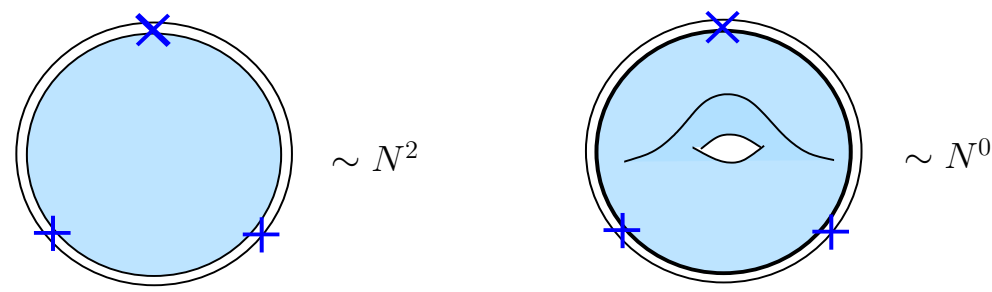

Figure 2: First order and second order contributions to the three meson interaction.

of flavors.

Here we will consider $N_{f}$ massless flavors and hence the Lagrangian has quantum global symmetry $S U\left(N_{f}\right)_{L} \times S U\left(N_{f}\right)_{R}$. The global chiral symmetry is expected to dynamically break to its maximal diagonal subgroup. For the Goldstone bosons of the theory one can now construct the associated chiral perturbation theory. The latter is an expansion in the number of derivatives acting on the Goldstone boson fields. The low energy effective Lagrangian describes the dynamics of the massless mesons that are the Nambu-Goldstone bosons of the spontaneous chiral symmetry breaking. Written in terms of the matrix $U(x)=\exp \left(\frac{i \pi(x)}{F_{\pi}}\right)$, where $\pi(x)$ is the Goldstone boson matrix, the effective Lagrangian is

$$
S_{\mathrm{eff}}=\frac{1}{16} F_{\pi}^{2} \int d^{4} x\left\{\operatorname{Tr}_{\mu} U \partial_{\mu} U^{-1}+\text { higher derivatives }\right\}+\Gamma_{\mathrm{WZW}} .
$$

The topological term, the Wess-Zumino-Witten (WZW) term, is essential in order to satisfy the t'Hooft anomaly conditions at the effective Lagrangian level. Gauging the WZW term with to respect the electromagnetic interactions yields the familiar $\pi^{0} \rightarrow 2 \gamma$ anomalous decay. The WZW term can be written as

$$
\Gamma_{\mathrm{WZW}}=-i \frac{n}{240 \pi^{2}} \int_{\mathcal{M}^{5}} \epsilon^{\mu \nu \rho \sigma \tau} \operatorname{Tr}\left(\partial_{\mu} U U^{-1} \partial_{\nu} U U^{-1} \partial_{\rho} U U^{-1} \partial_{\sigma} U U^{-1} \partial_{\tau} U U^{-1}\right) .
$$

where the integral must be performed over a five-dimensional manifold whose boundary is ordinary Minkowski space. Quantum consistency of the theory requires $n$ to 
be an integer. Matching with the underlying anomaly computations requires $n$ to be equal to the number of quarks with respect to the color that in the fundamental case is $N$. This imply that for the two index representation case $n=\frac{N(N \pm 1)}{2}$.

The low energy effective theory supports solitonic excitations which can be identified with the baryonic sector of the theory. In order to obtain classically stable configurations, it is necessary to include at least a four derivative term in addition to the usual two derivative term. Such a term can be for example the Skyrme term

$$
L_{\text {Skyrme }}=\frac{1}{32 e^{2}} \operatorname{Tr}\left(\left[\partial_{\mu} U U^{-1}, \partial_{\nu} U U^{-1}\right]^{2}\right),
$$

but what we will say is not dependent on the details of the higher-order terms. The Skyrmion is a texture-like solution of the effective Lagrangian arising from the non-trivial third homotopy group of the possible configurations of the matrix $U(x)$ (namely $\pi_{3}\left(S U\left(N_{f}\right)\right)=\mathbf{Z}$ ). In the large $N$ limit we can treat the effective Lagrangian as classical and, since the $N$ dependence appears only as a multiplicative factor, the size and the mass of the Skyrmion scale respectively as $N^{0}$ and $\frac{N(N \pm 1)}{2}$. This also implies that $e$ must scale as $1 / F_{\pi}$. Following [4] we can read off the statistics and the baryon number of the Skyrmion from the coefficient of the WZW term. The baryon number of the Skyrmion is $\frac{N(N \pm 1)}{2}$ the baryon number of the quarks and the statistics is fermionic or bosonic accordingly if $\frac{N(N \pm 1)}{2}$ is odd or even.

The results we have just obtained point all in the same direction. There should exist in the spectrum of the theory a stable baryon that in the large $N$ limit could be identified with the Skyrmion. This baryon should be constituted by $\frac{N(N \pm 1)}{2}$ quarks and its mass should scale like $N^{2}$ in the large $N$ limit.

\section{Baryons at Large $N$}

\subsection{The baryon in ordinary QCD}

Now we briefly review the large $N$ behavior of the baryon in ordinary QCD. The gauge wave function is

$$
\epsilon_{\alpha_{1} \ldots \alpha_{N}} Q^{\alpha_{1}} \ldots Q^{\alpha_{N}}
$$

and it is antisymmetric under exchange of two quarks. Since the quarks are fermions, the total gauge function $\psi_{\text {gauge }} \psi_{\text {spin/flavor }} \psi_{\text {space }}$ must be antisymmetric under exchange 
of two quarks. The simplest choice is to take a completely symmetric spin wave function and a completely symmetric spatial wave function.

$$
\begin{array}{ccc}
\psi_{\text {gauge }} & \psi_{\text {spin/flavor }} & \psi_{\text {space }} \\
- & + & +
\end{array}
$$

In the large $N$ limit the problem can be approximated by a system of free bosons in a mean field potential $V_{\text {mean }}(r)$ created by the quarks themseves. The ground state is a Bose-Einstein condensate; the quarks are all in the ground state of the mean field potential. The large $N$ behavior of the baryon is the following

$$
R \sim \mathcal{O}(1), \quad M \sim \mathcal{O}(N)
$$

where $R$ is the size of the baryon and $M$ its mass.

The key point to obtain this result is that the many body problem becomes enormously simplified by the fact that the coupling constant scales like $\frac{1}{g^{2}} \sim N$ in the large $N$ limit. To find the mass in this many body problem we have to sum up all the contributions from $k$-body interactions. The 1 -body contribution is simply $N$ times the mass of the single quark. The 2-body interaction is of order $\frac{1}{N}$ but an additional combinatorial factor $\left(\begin{array}{c}N \\ 2\end{array}\right)$ is needed and we obtain a contribution to the energy of order $N$. In general any $k$-body interaction is of order $\frac{1}{N^{k-1}}$ in the planar limit and multiplied by the combinatorial factor $\left(\begin{array}{c}N \\ k\end{array}\right)$ it gives a contribution of order $N$.

The same argument imply that the mean field potential $V_{\text {mean }}(r)$ is constant in the large $N$ limit and so also the typical size of baryon $R$. $R$ is in fact the with of the ground state wave function.

These arguments are consistent with the low-energy effective Lagrangian point of view. This Lagrangian is $L_{e f f} \sim N(\partial U \partial U+\partial U \partial U \partial U \partial U+\ldots)$ where $U$ is a $S U\left(N_{f}\right)$ matrix. Since $N$ is an overall multiplicative factor the radius of the Skyrmion is of order one while its mass is of order $N$.

\subsection{The simplest baryon in higher representations}

In higher representations QCD, the simplest baryon is

$$
\epsilon_{\alpha_{1} \alpha_{2} \ldots \alpha_{N}} \epsilon_{\beta_{1} \beta_{2} \ldots \beta_{N}} Q^{\left\{\alpha_{1} \beta_{1}\right]} Q^{\left\{\alpha_{2} \beta_{2}\right]} \ldots Q^{\left\{\alpha_{N} \beta_{N}\right]} .
$$

If we exchange two quarks, say for example $Q^{\left\{\alpha_{1} \beta_{1}\right]}$ and $Q^{\left\{\alpha_{2} \beta_{2}\right]}$, this is equivalent to the exchange of $\alpha_{1} \alpha_{2}$ in $\epsilon_{\alpha_{1} \alpha_{2} \ldots \alpha_{N}}$ and $\beta_{1} \beta_{2}$ in $\epsilon_{\beta_{1} \beta_{2} \ldots \beta_{N}}$. The result is that the gauge 
wave function is symmetric under exchange of two quarks. This means that in order to have a total wave function that is antisymmetric under exchange, the spatial wave function $\psi_{\text {space }}$ must be antisymmetric.

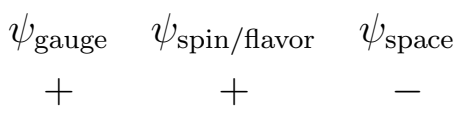

In the large $N$ limit the problem can be approximated by a system of free fermions in a mean field potential $V_{\text {mean }}(r)$. The ground state is a degenerate Fermi gas and is obtained by filling all the lowest energy states of the mean field potential up the Fermi surface. Now there are two kind of forces that enter in the game:

1) Gauge forces scales like $N$ and are both repulsive and attractive,

2) Fermi zero temperature pressure scales like $N^{4 / 3}$ and is only repulsive 2

We can thus immediately infer the following that the simplest baryon cannot be matched with the Skyrmion; this is because the mass of the Skyrmion gous like $N^{2}$ while the mass of this baryon obviously cannot go faster than $N^{4 / 3}$.

In the next Section we shall construct the baryon that must be identifyed with the Skyrmion of the low-energy effective Lagrangian. In Section 6 we shall see that this baryon, although heavier than the simplest one, is generally stable and cannot decay.

\section{The Skyrmion in the Fundamental Theory}

We have seen in the previous section that the simplest baryon has a gauge wave function which is symmetric under exchange of two quarks. This has a drastic consequence on its mass vs. $N$ dependence in the large $N$ limit. In the following we will construct the only possible gauge wave function that is completely antisymmetric under exchange of two quarks. We will find that the required number of quarks, as expected from the Skyrmion analysis, is $\frac{N(N \pm 1)}{2}$.

\footnotetext{
${ }^{2}$ An important thing to note is that in the large $N$ limit we always reach an ultrarelativistic regime due to the fact that the energy at the Fermi surface goes to infinity as $N$ goes to infinity.
} 


\subsection{The symmetric representation}

We start from the simplest case: $N=2$. We want to construct a gauge invariant wave function that contains three quarks $Q^{\left\{\alpha_{1} \beta_{1}\right\}}, Q^{\left\{\alpha_{2} \beta_{2}\right\}}$, and $Q^{\left\{\alpha_{3} \beta_{3}\right\}}$ that are antisymmetric under exchange of two quarks. First of all we put the three quarks in a triangular diagram like in Figure 3. Then we "formally" split the high-representational

$$
\begin{gathered}
Q^{\left\{\alpha_{1} \beta_{1}\right\}} \\
Q^{\left\{\alpha_{2} \beta_{2}\right\}} \quad Q^{\left\{\alpha_{3} \beta_{3}\right\}}
\end{gathered}
$$

Figure 3: The three quarks are placed in a triangular graphic.

quarks into two fundamental quarks. For example the quark $Q^{\left\{\alpha_{1} \beta_{1}\right\}}$ is splitted into two fundamental quarks $q^{\alpha_{1}}$ and $q^{\beta_{1}}$. The diagram of Figure 3 is doubled and the result is Figure 4 where we have respect the reflection symmetry with respect to the dashed line. Then we make a translation of the lower triangle so that the diagram

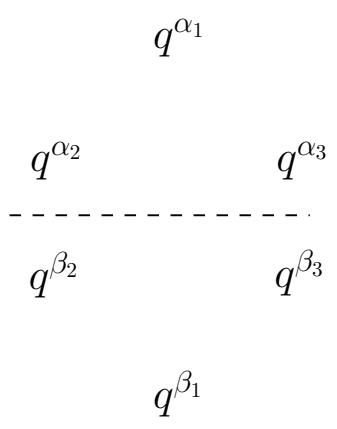

Figure 4: The quarks $Q^{\alpha \beta}$ are formally splitted in two fundamental quarks $q^{\alpha}$ and $q^{\beta}$.

becomes that of Figure 5. Finally we saturate the indices with three antisymmetric tensors $\epsilon$ that are the gray lines in Figure [5. The gauge wave function that correspond to the diagram is thus 3

$$
\epsilon_{\alpha_{2} \alpha_{1}} \epsilon_{\beta_{2} \alpha_{3}} \epsilon_{\beta_{1} \beta_{3}} Q^{\left\{\alpha_{1} \beta_{1}\right\}} Q^{\left\{\alpha_{2} \beta_{2}\right\}} Q^{\left\{\alpha_{3} \beta_{3}\right\}} .
$$

\footnotetext{
${ }^{3}$ Note also that the symmetric representation for $S U(2)$ is equivalent to the adjoint representation and a gauge invariant antisymmetric wave function can easily be written as $\epsilon_{a b c} Q^{a} Q^{b} Q^{c}$ where $a, b, c$ are triplet indices. This wave function is exactly the same as that of Eq. (4.1).
} 


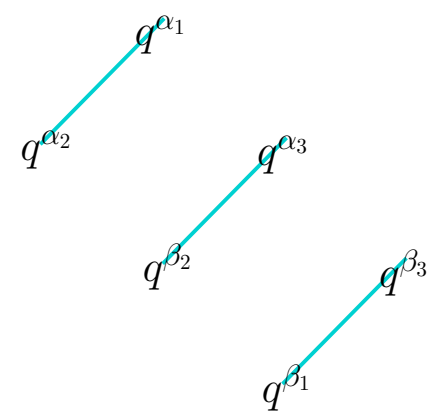

Figure 5: Diagrammatic representation of the baryon for $N=2$.

To prove that this wave function is antisymmetric under exchange of two quarks we can proceed in two ways: algebraic and diagrammatic. The algebraic proof is:

$$
\begin{array}{r}
\quad \epsilon_{\alpha_{1} \alpha_{2}} \epsilon_{\beta_{1} \alpha_{3}} \epsilon_{\beta_{2} \beta_{3}} Q^{\left\{\alpha_{1} \beta_{1}\right\}} Q^{\left\{\alpha_{2} \beta_{2}\right\}} Q^{\left\{\alpha_{3} \beta_{3}\right\}} \\
=-\epsilon_{\alpha_{2} \alpha_{1}} \epsilon_{\beta_{1} \alpha_{3}} \epsilon_{\beta_{2} \beta_{3}} Q^{\left\{\alpha_{1} \beta_{1}\right\}} Q^{\left\{\alpha_{2} \beta_{2}\right\}} Q^{\left\{\alpha_{3} \beta_{3}\right\}} \\
=-\epsilon_{\alpha_{2} \alpha_{1}} \epsilon_{\beta_{1} \beta_{3}} \epsilon_{\beta_{2} \alpha_{3}} Q^{\left\{\alpha_{1} \beta_{1}\right\}} Q^{\left\{\alpha_{2} \beta_{2}\right\}} Q^{\left\{\beta_{3} \alpha_{3}\right\}} \\
=-\epsilon_{\alpha_{2} \alpha_{1}} \epsilon_{\beta_{1} \beta_{3}} \epsilon_{\beta_{2} \alpha_{3}} Q^{\left\{\alpha_{1} \beta_{1}\right\}} Q^{\left\{\alpha_{2} \beta_{2}\right\}} Q^{\left\{\alpha_{3} \beta_{3}\right\}}
\end{array}
$$

The three passages are:

$(\mathrm{A} \rightarrow \mathrm{B})$ Exchange of $\alpha_{1}$ and $\alpha_{2}$ in the $\epsilon$ that brings a minus factor,

$(\mathrm{B} \rightarrow \mathrm{C})$ Renomination of $\alpha_{3}$ with $\beta_{3}$ which has no consequences,

$(\mathrm{C} \rightarrow \mathrm{D})$ Exchange of $\alpha_{3}$ and $\beta_{3}$ in the quark also has no consequences.

The diagrammatic proof is give in Figure 6. Note the correspondence between the three passages of the algebraic proof and the three passages of the diagrammatic proof. Before going on to higher $N$ we prove a general theorem that will also give us the recepie to build the desired gauge wave function.

Proposition 1 There is one and only one gauge wave function that is a gauge singlet and completely antisymmetric under exchange of two quarks. This wave function is composed by $\frac{N(N+1)}{2}$ quarks $Q^{\{\alpha \beta\}}$ and is the completely antisymmetric subspace of the tensor product of $\frac{N(N+1)}{2}$ quarks $Q^{\{\alpha \beta\}}$. 

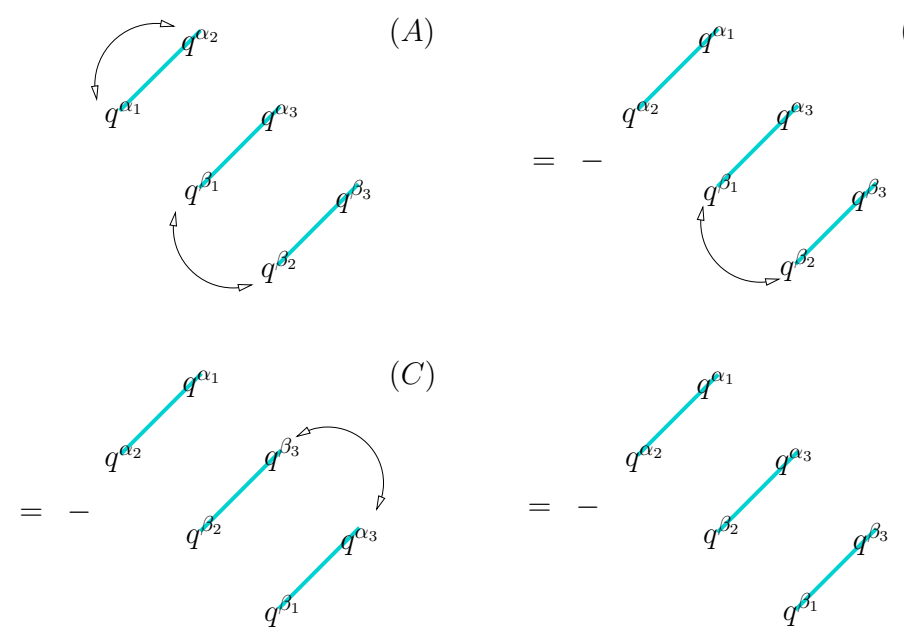

$(D)$

Figure 6: Diagrammatic proof that the baryon of Eq. (4.1) is antisymmetric under exchange of two quarks. The various steps $(A),(B),(C)$ and $(D)$ correspond to the passages of the algebraic proof (4.2).

Proof. Call $S$ the number of quarks in a hypothetical gauge wave function that satisfies the previous conditions. We can split the quarks $Q^{\{\alpha \beta\}}$ into $2 S$ fundamental quarks $q^{\alpha_{1}} \ldots q^{\alpha_{M}}$ and $q^{\beta_{1}} \ldots q^{\beta_{M}}$. The wave function is the sum of various pieces 4 where every piece is the partition of the $2 S$ fundamental quarks in sets of $N$ elements. These sets correspond to the saturation with the $\epsilon$ contraction and we indicate them with a red line that connects $N$ fundamental quarks. We need two facts to prove the proposition: 1) $q^{\alpha_{i}}$ and $q^{\beta_{i}}$ cannot belong to the same saturation line since the indices $\alpha_{i}$ and $\beta_{i}$, belongings to the same quark $Q^{\left\{\alpha_{i} \beta_{i}\right\}}$, are symmetric under exchange; 2) If $q^{\alpha_{i}}$ and $q^{\alpha_{j}}$ belong to the same saturation line, the two partners $q^{\beta_{i}}$ and $q^{\beta_{j}}$ cannot belong to the same saturation line. The reason is simply that an exchange of $Q^{\left\{\alpha_{i} \beta_{i}\right\}}$ and $Q^{\left\{\alpha_{j} \beta_{j}\right\}}$ would give a plus sign instead of the required minus sign. At this point we are ready to draw the diagram of Figure 7 that is needed for the proof. We first draw the first saturation line containing the fundamental quarks. Due to 1) they cannot belong to the same quark and so we can call them $q^{\alpha_{1}} \ldots q^{\alpha_{N}}$. Any of these fundamental quarks must have a partner $q^{\beta_{1}} \ldots q^{\beta_{N}}$ and we draw them on the diagram. Due to 2), any of the last quarks must belong to a different saturation line.

\footnotetext{
${ }^{4}$ For the wave function of $\frac{N(N+1)}{2}$ quarks there is only one piece that corresponds to one diagram. In principle there could be more terms added together. This in fact is the case when we consider the antisymmetric representation.
} 
So, only starting from one saturation line, we have shown the existence of at least

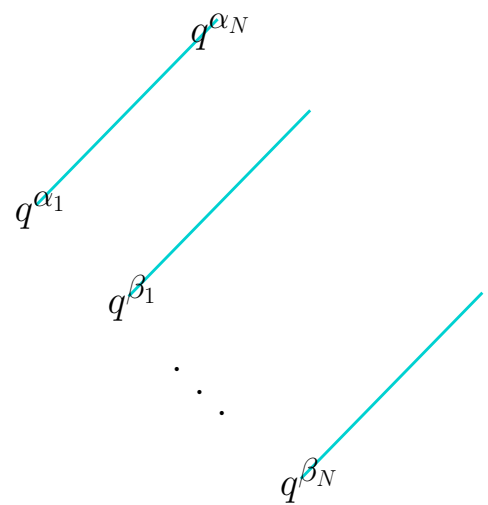

Figure 7: Diagrammatic proof that the minimum number of quarks to form an antisymmetric baryon is $\frac{N(N+1)}{2}$.

$N(N+1)$ fundamental quarks in the diagram. This implies that $S \geq \frac{N(N+1)}{2}$.

Now we need to prove the existence of this wave function. Consider the tensor product of certain number of quarks $Q^{\{\alpha \beta\}}$. Every quark must be considered as a vector space of dimension $\frac{N(N+1)}{2}$ over which the group $S U(N)$ act as a linear representation. Now we take the subspace of the tensor product that is completely antisymmetric under exchange. This subspace is obviously closed under the action of the gauge group. If the number of quarks is greater than $\frac{N(N+1)}{2}$ this subspace has dimension zero. If the number of quarks is exactly $\frac{N(N+1)}{2}$ the antisymmetric subspace has dimension one. We have thus proven that the completely antisymmetric space of $\frac{N(N+1)}{2}$ quarks $Q^{\{\alpha \beta\}}$ is also a singlet of the gauge group.

The gauge wave function for general $N$ can be obtained by generalizing the one of Figure 5 for $N=2$. For example the diagram for $N=3$ is given in Figure 8 .

The baryon for $N=2$ of Figure 5 does not need to be antisymmetrized because it is already antisymmetric under exchange of any pair of quarks. For $N=3$ the antisymmetrizations with respect to the four quarks $Q^{\left\{\alpha_{1} \beta_{1}\right\}}, Q^{\left\{\alpha_{2} \beta_{2}\right\}}, Q^{\left\{\alpha_{3} \beta_{3}\right\}}$ and $Q^{\left\{\alpha_{4} \beta_{4}\right\}}$ is enough to guarantee the complete antisymmetrization. From the diagram in Figure 8 it can be seen that the antisymmetrization with respect to the exchange $Q^{\left\{\alpha_{1} \beta_{1}\right\}} \leftrightarrow Q^{\left\{\alpha_{2} \beta_{2}\right\}}$ implies that with respect to $Q^{\left\{\alpha_{3} \beta_{3}\right\}} \leftrightarrow Q^{\left\{\alpha_{5} \beta_{5}\right\}}$ and the same for the two exchanges $Q^{\left\{\alpha_{2} \beta_{2}\right\}} \leftrightarrow Q^{\left\{\alpha_{4} \beta_{4}\right\}}$ and $Q^{\left\{\alpha_{3} \beta_{3}\right\}} \leftrightarrow Q^{\left\{\alpha_{6} \beta_{6}\right\}}$. We have thus the sufficient amount of exchanges to generate the complete permutation group. 


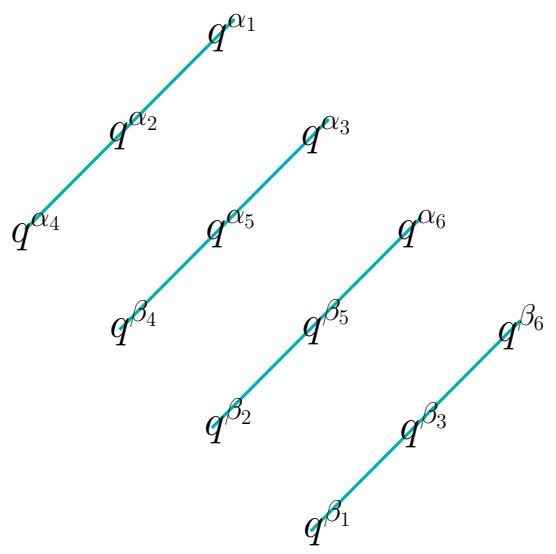

Figure 8: The diagram for $N=3$.

\subsection{The antisymmetric representation}

As we have done in the previous subsection we start with simplest cases and then we try to generalize. Our goal is a gauge invariant and antisymmetric wave function that contains $\frac{N(N-1)}{2}$ quarks $Q^{[\alpha \beta]}$. For $N=2$, we have $\frac{N(N-1)}{2}=1$, and it is easy to find such a wave function $\epsilon_{\gamma \delta} Q^{[\gamma \delta]}$. For $N=3$ we need a wave function that contains three quarks. To guess it using directly $Q^{[\alpha \beta]}$ is not easy, but we can use a trick. The antisymmetric representation for $N=3$ is equivalent to the anti-fundamental $\widetilde{Q}_{\gamma}=\frac{1}{2} \epsilon_{\gamma \alpha \beta} Q^{[\alpha \beta]}$ and we know how to write a baryon for the antifundamental representation

$$
\epsilon^{\gamma \rho \tau} \widetilde{Q}_{\gamma} \widetilde{Q}_{\rho} \widetilde{Q}_{\tau}
$$

Substituting the relation between $\widetilde{Q}_{\gamma}$ and $Q^{[\alpha \beta]}$ we obtain

$$
\frac{1}{2}\left(\epsilon_{\gamma_{1} \delta_{1} \alpha} \epsilon_{\gamma_{2} \delta_{2} \beta}-\epsilon_{\gamma_{2} \delta_{2} \alpha} \epsilon_{\gamma_{1} \delta_{1} \beta}\right) Q^{[\alpha \beta]} Q^{\left[\gamma_{1} \delta_{1}\right]} Q^{\left[\gamma_{2} \delta_{2}\right]} .
$$

We know by construction that this wave function is antisymmetric under exchange of any couple of quarks. Before going on we prove a general theorem.

Proposition 2 There is one and only one gauge wave function that is gauge singlet and completely antisymmetric under exchange of two quarks. This wave function is composed by $\frac{N(N-1)}{2}$ quarks $Q^{[\alpha \beta]}$ and is the antisymmetric subspace of the tensor product of $\frac{N(N-1)}{2}$ quarks $Q^{[\alpha \beta]}$.

Proof. Denote by $A$ the number of quarks in a hypothetical gauge wave function that satisfies the previous conditions. The reason why $A$ can be smaller than $S$ is 
that now it is instead possible for a quark to have both indices on the same saturation line. By convention we will denote these quarks by $Q^{[\gamma \delta]}$ while the other ones, whose indices belong to different saturation lines, will be denoted as before $Q^{[\alpha \beta]}$. Only the quarks of the type $Q^{[\alpha \beta]}$ will be splitted into two fundamental quarks $q^{\alpha}$ and $q^{\beta}$. For the proof we need the following two basic facts: 1) One saturation line can contain at most one quark of the type $Q^{\left[\gamma_{i} \delta_{i}\right]}$ otherwise the wave function will be symmetric under exchange of these quarks. 2) If $q^{\alpha_{i}}$ and $q^{\alpha_{j}}$ belong to the same saturation line, the two partners $q^{\beta_{i}}$ and $q^{\beta_{j}}$ cannot belong to the same saturation line. The reason is the same as in the case of the symmetric representation. At this point we are ready to draw the diagram of Figure 9 that is needed for the proof. We first draw the first saturation line that contain one quark $Q^{\left[\gamma_{1} \delta_{1}\right]}$ and $N-2$ fundamental quarks $q^{\alpha_{1}} \ldots q^{\alpha_{N-2}}$. In principle we could also have zero quarks of the type $Q^{[\gamma \delta]}$ on one saturation line but, since we want to minimize the number of quarks in the wave function, we will assume that every saturation line contains one and only one quark of the type $Q^{[\gamma \delta]}$. Due to 2$)$, the partners of $q^{\alpha_{1}} \ldots q^{\alpha_{N-2}}$, that we denote by $q^{\beta_{1}} \ldots q^{\beta_{N-2}}$, must belong to different saturation lines and any of these lines will contain one quark $Q^{\left[\gamma_{2} \delta_{2}\right]} \ldots Q^{\left[\gamma_{N-1} \delta_{N-1}\right]}$.

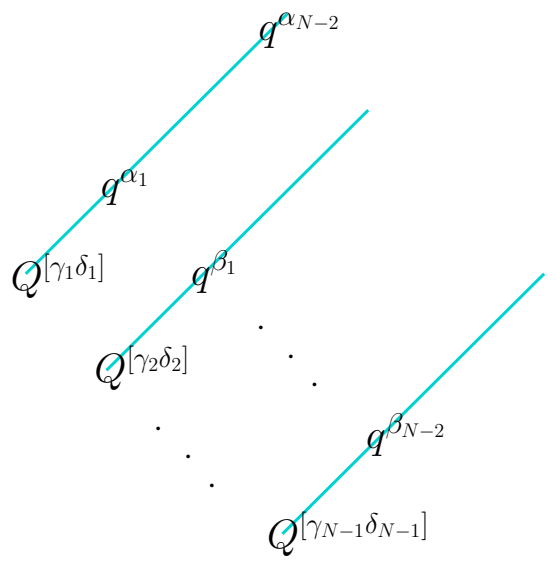

Figure 9: Diagrammatic proof that the minimum number of quarks to form an antisymmetric baryon is $\frac{N(N-1)}{2}$.

The proof of the existence is exactly the same as that of the symmetric representation.

The wave function for $N=3$ is Eq. (4.4) and has already been written respecting the conventions pointed out in the proof. Now we are ready to write the diagram. 
First of all there are two terms and so there will be two diagrams. Every diagram will be divided in two parts. On one side we put the quarks $Q^{\left[\gamma_{1} \delta_{1}\right]}, Q^{\left[\gamma_{2} \delta_{2}\right]}$ and on the other side the fundamental quarks $q^{\alpha}$ and $q^{\beta}$. Finally we draw the saturation lines and the two diagrams (with the needed signs) are shown in Figure10. For example the baryon
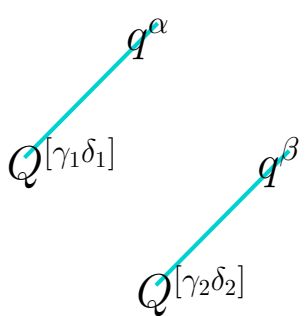

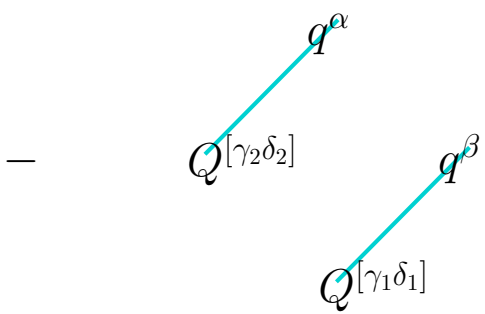

Figure 10: Diagrammatic representation of the baryon for $N=3$.

for $N=4$ is given by the diagram in Figure 11 plus the needed antisymmetrizations. For convenience the quarks $Q^{\gamma_{1} \delta_{1}}, Q^{\gamma_{1,2,3} \delta_{1,2,3}}$ are now called $Q^{\alpha_{4,5,6} \delta_{4,5,6}}$. The gauge

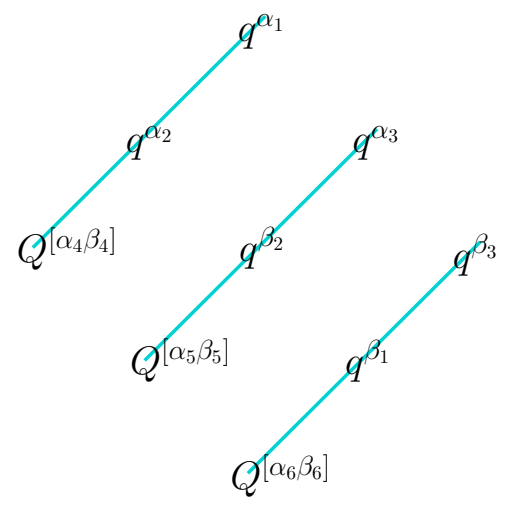

Figure 11: The diagram for $N=4$.

wave function is

$$
\begin{aligned}
& \left(\sum_{\sigma \in S} \operatorname{sign}(\sigma) \epsilon_{\alpha_{\sigma(4)} \beta_{\sigma(4)} \alpha_{\sigma(2)} \alpha_{\sigma(1)} \epsilon_{\sigma(2)} \beta_{\sigma(5)} \beta_{\sigma(2)} \alpha_{\sigma(3)}} \epsilon_{\alpha_{\sigma(6)} \beta_{\sigma(6)} \beta_{\sigma(1)} \beta_{\sigma(3)}}\right) \\
& Q^{\left\{\alpha_{1} \beta_{1}\right\}} Q^{\left\{\alpha_{2} \beta_{2}\right\}} Q^{\left\{\alpha_{3} \beta_{3}\right\}} Q^{\left\{\alpha_{4} \beta_{4}\right\}} Q^{\left\{\alpha_{5} \beta_{5}\right\}} Q^{\left\{\gamma_{3} \delta_{3}\right\}} .
\end{aligned}
$$




\section{More on the Antisymmetric Representation: a Large $N$ Limit for QCD}

In this section we want to consider in more detail the case of the antisymmetric representation, in particular its importance in describing a large $N$ limit for QCD.

The simplest baryon previously considered

$$
\epsilon_{\alpha_{1} \alpha_{2} \ldots \alpha_{N}} \epsilon_{\beta_{1} \beta_{2} \ldots \beta_{N}} Q^{\left[\alpha_{1} \beta_{1}\right]} Q^{\left[\alpha_{2} \beta_{2}\right]} \ldots Q^{\left[\alpha_{N} \beta_{N}\right]}
$$

must be carefully reanalyzed in the case of the antisymmetric representation. We have now to make a distinction between $N$ even and $N$ odd. In the case of $N$ even (5.1) is not the minimal baryon since we can construct a gauge invariant wave function using only $N / 2$ quarks:

$$
\epsilon_{\gamma_{1} \gamma_{2} \ldots \gamma_{N / 2} \delta_{1} \delta_{2} \ldots \delta_{N / 2}} Q^{\left[\gamma_{1} \delta_{1}\right]} Q^{\left[\gamma_{2} \delta_{2}\right]} \ldots Q^{\left[\gamma_{N / 2} \delta_{N / 2}\right]}
$$

This baryon is symmetric under exchange of two quarks and so there is no difference with respect to the previous conclusion: in the large $N$ limit its mass is asymptotically proportional to $N^{4 / 3}$ and so it is much heavier than the baryon constructed with $\frac{N(N-1)}{2}$ quarks.

The case of $N=2 n+1$ odd is more interesting. We can prove that the minimal baryon (5.1) is identically zero with the following algebraic passages:

$$
\begin{array}{r}
\epsilon_{\alpha_{1} \alpha_{2} \ldots \alpha_{2 n+1}} \epsilon_{\beta_{1} \beta_{2} \ldots \beta_{2 n+1}} Q^{\left[\alpha_{1} \beta_{1}\right]} Q^{\left[\alpha_{2} \beta_{2}\right]} \ldots Q^{\left[\alpha_{2 n+1} \beta_{2 n+1}\right]} \\
=(-1)^{2 n+1} \epsilon_{\alpha_{1} \alpha_{2} \ldots \alpha_{2 n+1}} \epsilon_{\beta_{1} \beta_{2} \ldots \beta_{2 n+1}} Q^{\left[\beta_{1} \alpha_{1}\right]} Q^{\left[\beta_{2} \alpha_{2}\right]} \ldots Q^{\left[\beta_{2 n+1} \alpha_{2 n+1}\right]} \\
=\quad-\epsilon_{\beta_{1} \beta_{2} \ldots \beta_{2 n+1}} \epsilon_{\alpha_{1} \alpha_{2} \ldots \alpha_{2 n+1}} Q^{\left[\alpha_{1} \beta_{1}\right]} Q^{\left[\alpha_{2} \beta_{2}\right]} \ldots Q^{\left[\alpha_{2 n+1} \beta_{2 n+1}\right]} .
\end{array}
$$

In the first passage we have exchanged the $\alpha$ and the $\beta$ indices in every quark. Since we have $2 n+1$ quarks in the antisymmetric representation this step brings down a minus sign. In the second step we have just renamed $\alpha_{i}$ with $\beta_{i}$ and vice versa and this has no consequences. The last line of (5.3) is equal to minus the fist line (a part from an irrelevant exchange in the position of the two epsilons) and thus the wave function must be zero. We will now prove a stronger statement:

Proposition 3 For $N$ odd and quarks in the antisymmetric representation, it is not possible to write a gauge invariant wave function that is completely symmetric under exchange of two quarks. 
Proof. Take a generic wave function that is gauge invariant and symmetric under exchange of two quarks. We are going to prove that it is identically zero. This wave function is composed by a number of quarks that we generically denote by $M . M_{\alpha \beta}$ of these quarks are of type $Q^{[\alpha \beta]}$ and $M_{\gamma \delta}$ are of type $Q^{[\gamma \delta]}$ so that we can write

$$
M=M_{(\alpha \beta)}+M_{(\gamma \delta)} .
$$

The $M$ quarks can be divided into various connected components, where the connection is given by the epsilon contractions and the quarks $Q^{[\alpha \beta]}$. Let us assume for the moment that we have only one connected component. It is easy to see that $M_{\alpha \beta}$ must be odd. We will now use the same argument we have used to show that (5.3) is identically zero. Namely we will show that the wave function is equal to minus itself. first we exchange all the $\alpha$ indices with their $\beta$ partners and this contributes a minus sign since $M_{\alpha \beta}$ is odd. Then we make a suitable number of exchange between the quarks $Q^{[\gamma \delta]}$ in order to recover the original epsilon structure. These exchanges do not affect the wave function since by definition it is symmetric under exchanges of two quarks. So we have recovered the original wave function but with a minus sign in front.

We now have to consider the more general situation in which the $M$ quarks are divided in various disconnected components. It can easily be seen that in this case the sub-connected components must be closed under the exchange of two generic quarks. Put in another way, if the global wave function is symmetric under exchange of two quarks, then also the sub-connected wave functions are symmetric under exchange of two quarks. In this case we can thus remake the passage of the previous paragraph but only on a sub-connected wave function and we obtain the desired result.

The previous proposition does not exclude the possible existence of a gauge invariant wave function with less then $\frac{N(N-1)}{2}$ quarks and in a non singlet representation of the permutation group. In this case the baryon is not a simple product of gauge, spin and space wave function but a sum $\sum_{i} \psi_{\text {gauge }}^{i} \psi_{\text {spin }}^{i} \psi_{\text {space }}^{i}$, where $\psi_{\text {gauge }}^{i}$ is the non-singlet representation of the permutation group.

\section{$6 \quad$ Stability of the Skyrmion}

We want now to discuss the issue of the stability of the Skyrmion. The Skyrmion correspond to the baryon that contains $\frac{N(N \pm 1)}{2}$ quarks and that is fully antisymmetric 
in the gauge wave function. The mass is thus proportional to the number of costituents quarks. Seen from the low-energy effective Lagrangian, the Skyrmion is absolutly stable. In the full theory, on the other hand, we should consider the possibility of decay into baryons with lower numbers of costituents quarks, for example the baryon $\epsilon_{\alpha_{1} \alpha_{2} \ldots \alpha_{N}} \epsilon_{\beta_{1} \beta_{2} \ldots \beta_{N}} Q^{\left\{\alpha_{1} \beta_{1}\right]} Q^{\left\{\alpha_{2} \beta_{2}\right]} \ldots Q^{\left\{\alpha_{N} \beta_{N}\right]}$. This states are not visible from the lowenergy effective Lagrangian. As we have seen in Section 4 baryons with a number of costituents quarks lower than $\frac{N(N \pm 1)}{2}$ can not be in a fully antisymmetric gauge wave function. This imply that the Skyrmion is the state that minimizes the mass per unit of baryon number.

Let us consider an explicit example in more detail. A Skyrmion that contains $\frac{N(N \pm 1)}{2}$ can decay into $\frac{N \pm 1}{2}$ baryons composed by $N$ quarks. The baryon number is conserved and so this decay channel is in principle possiple. In order to analize the energetic of this baryon, we propose now a toy model to schematize the fundamental baryon. We have $N$ quarks and 2 baryon vertices. Every quark is attached to two fundamental strings and every baryon vertex to $N$ fundamental strings (see Figure 12 for an example). Baryon vertices have a mass of order $N$; we can thus neglect their

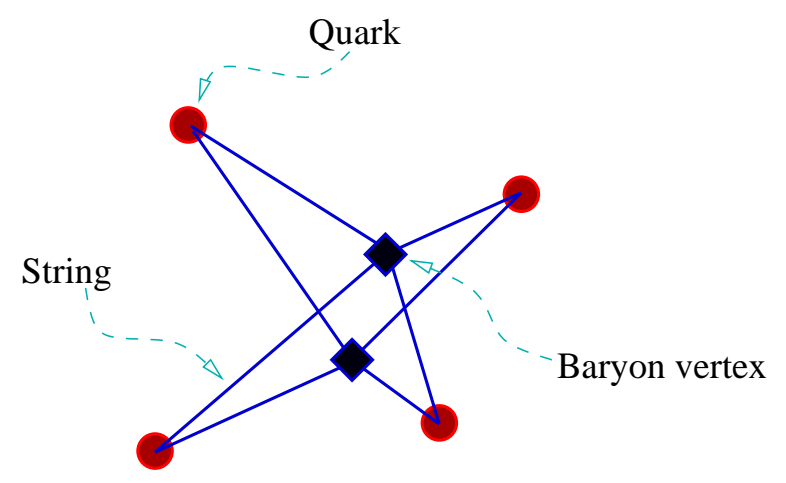

Figure 12: A model of the baryon (here for four colors). Every quark is attached to two confining strings and every baryon vertex to $N$ confining strings.

dynamics and consider them at rest and positioned in what we define to be the center of the baryon. In this approximation, the quarks do not interact directly between each other; they live in a mean potential given by the string tension multiplyed by the distance from the center

$$
V_{\text {mean }}(R)=2 T_{\text {string }}|R| \text {. }
$$


Quarks are antysymmetric in the space wave function and so they they fill the energy levels up to the Fermi surface (see Figure 13). We indicate as $R_{\mathrm{F}}$ and $P_{\mathrm{F}}$ respectively the Fermi radius and momentum. The total energy and the number of quarks $N$ are given by the following integrals over the phase space:

$$
\begin{array}{r}
\int^{R_{\mathrm{F}}} \int^{P_{\mathrm{F}}} \frac{d^{3} R d^{3} P}{(2 \pi)^{3}}\left(P+V_{\text {mean }}(R)\right)=E, \\
\int^{R_{\mathrm{F}}} \int^{P_{\mathrm{F}}} \frac{d^{3} R d^{3} P}{(2 \pi)^{3}}=N .
\end{array}
$$

Since the quarks are massless we take the Hamiltonian to be $P+V_{\text {mean }}(R)$. From now on we neglect numerical factors such as the phase space volume element, at this level of approximation they are not important. The second equation of (6.2) gives a relation between the Fermi momentum and the Fermi radius, namely $P_{\mathrm{F}} \sim N^{1 / 3} / R_{\mathrm{F}}$. The first equation of (6.2) gives the following expression of the energy as function of the radius

$$
E \sim \frac{N^{4 / 3}}{R_{\mathrm{F}}}+T_{\text {string }} N R_{\mathrm{F}}
$$

Minimizing we obtain $R_{\mathrm{F}} \sim N^{1 / 6} / \sqrt{T_{\text {string }}}$, and consequently $P_{\mathrm{F}} \sim N^{1 / 6} \sqrt{T_{\text {string }}}$. The mass of the baryon is thus given by

$$
M_{N-\text { Baryon }} \sim N^{7 / 6} \sqrt{T_{\text {string }}} .
$$

The important thing to note is the $N^{7 / 6}$ dependence. The mass per unit of baryon number grows as $N^{1 / 6}$. The Skyrmion has instead mass per unit of baryon number of order one. This imply that the Skyrmion is the most convenient baryonic state since it minimizes the energy per unit of baryon number.

This approximation breaks down when the Fermi energy $N^{1 / 6} \sqrt{T_{\text {string }}}$ becomes much grater than the dynamical scale. Due to asymptotic freedom, the highly energetic quarks do not feel a confining potential like (6.1) but instead a Coulomb-like potential. The mass per unit of barion number stops to grow as $N^{1 / 6}$ and saturates to a constant.

\section{Acknowledgements}

I thank especially F. Sannino for the suggestion to work on this problem and for the many useful discussions. I thank S. B. Gudnason for discussions and for the precious 


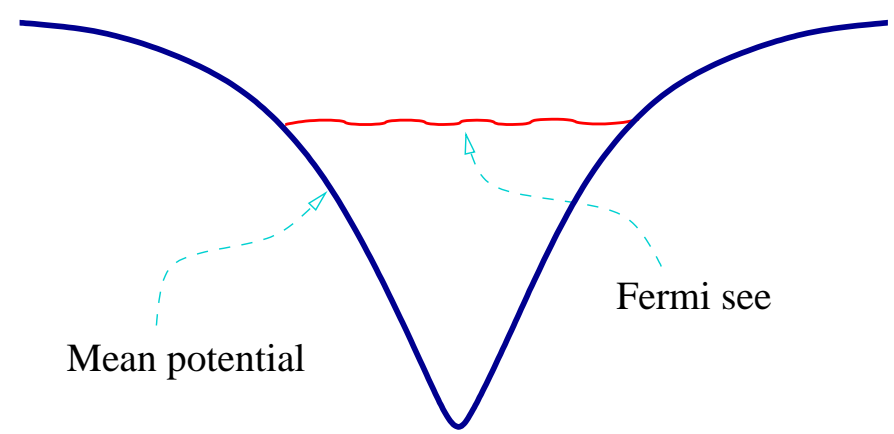

Figure 13: The mean potential for our toy model of the baryon.

help with the manuscript. The present work has been presented at the conference CAQCD in Minneapolis. I want to thank M. Shifman and T. Cohen for interesting observations. This work is supported by the Marie Curie Excellence Grant under contract MEXT-CT-2004-013510.

\section{References}

[1] G. 't Hooft, "A Planar Diagram Theory For Strong Interactions," Nucl. Phys. B 72 (1974) 461.

[2] E. Witten, "Baryons In The 1/N Expansion," Nucl. Phys. B 160 (1979) 57.

[3] T. H. R. Skyrme, "A Nonlinear Field Theory," Proc. Roy. Soc. Lond. A 260 (1961) 127.

[4] E. Witten, "Current Algebra, Baryons, And Quark Confinement," Nucl. Phys. B 223 (1983) 433.

[5] J. Wess and B. Zumino, "Consequences Of Anomalous Ward Identities," Phys. Lett. B 37 (1971) 95.

[6] E. Witten, "Global Aspects Of Current Algebra," Nucl. Phys. B 223 (1983) 422.

[7] G. Veneziano, "Some Aspects Of A Unified Approach To Gauge, Dual And Gribov Theories," Nucl. Phys. B 117, 519 (1976). 
[8] E. Corrigan and P. Ramond, "A Note On The Quark Content Of Large Color Groups," Phys. Lett. B 87, 73 (1979).

[9] A. Armoni, M. Shifman and G. Veneziano, "Exact results in nonsupersymmetric large N orientifold field theories," Nucl. Phys. B 667, 170 (2003) |arXiv:hep-th/0302163]; "Refining the proof of planar equivalence," Phys. Rev. D 71, 045015 (2005) arXiv:hep-th/0412203.

[10] A. Armoni, M. Shifman and G. Veneziano, "From super-Yang-Mills theory to QCD: Planar equivalence and its implications," arXiv:hep-th/0403071.

[11] A. Armoni, M. Shifman and G. Veneziano, "SUSY relics in one-flavor QCD from a new 1/N expansion," Phys. Rev. Lett. 91, 191601 (2003) [arXiv:hep-th/0307097].

[12] A. Armoni and M. Shifman, "The cosmological constant and domain walls in orientifold field theories and N = 1 gluodynamics," Nucl. Phys. B 670 (2003) 148 arXiv:hep-th/0303109. 